\title{
An Infrared Colour Discriminator for Giant and Supergiant Optical M Stars
}

\author{
Moedji Raharto \\ Bosscha Observatory and Department of Astronomy, Bandung Institute \\ of Technology, Indonesia
}

\begin{abstract}
An infrared colour - MK spectral type diagram of optical $M$ giants, $M$ Miras and $M$ supergiants is presented. The area in the diagram with $m_{12}-m_{25}>0.70$ and MK spectral type earlier than M4 is occupied by early type optical $M$ supergiants. The region of spectral type later than M4 is occupied by optical late M giants (Mira variables) and late $M$ supergiants. The area in the diagram with a colour range of $0.15<m_{12}-m_{25}<0.70$ is occupied by optical giants (Mira Variables) and supergiants ( $M$ supergiants with blue companions and probably some $M$ supergiants in the evolutionary stage pre or post $M$ supergiants with large infrared excesses). Finally, the area in the diagram with $m_{12}-m_{25}<$ 0.15 is clearly occupied by $M$ giants without circumstellar dust shells. The colour distribution of the $M$ giants, $M$ supergiants and $M$ Miras in the colour - spectral type diagram suggests that we can use the colour properties as search criteria for (i) early $M$ supergiants with colours of $m_{12}-m_{25}>0.70$, (ii) late M supergiants with colours of $m_{12}-m_{25}>0.70$, (iii) $M$ giants with $m_{12}-m_{25}<0.15$, (iv) Mira candidates using IRAS point sources which are also identified as optical M stars.
\end{abstract}

\section{Introduction}

Low resolution spectroscopic surveys in the near-infrared $(\lambda 6800-8800 \AA)$ using Schmidt telescopes equipped with low dispersion (typically $700-1870 \AA / \mathrm{mm}$ at A band) objective prisms provide a powerful deep survey method for searching for galactic $M$ stars in large sky regions. Examples of such optical surveys for galactic M stars are given by Mavridis (1971), Albers (1972), Ichikawa et al. (1982) and MacConnell et al. (1987). The infrared sky survey by IRAS (InfraRed Astronomical Satellite) in 1983 provided homogeneous infrared photometry of galactic infrared point sources at $12,25,60$ and $100 \mu \mathrm{m}$ for most of the sky. Based on the results of various studies of galactic infrared point sources identified as M giants or M supergiants (e.g. Hacking et al. 1985; Ishida \& Othman 1988 ), it can be concluded that galactic optical M giants, Mira variables and $M$ supergiants are among the bright infrared sources. Many of these stars were indeed detected during the IRAS survey. 


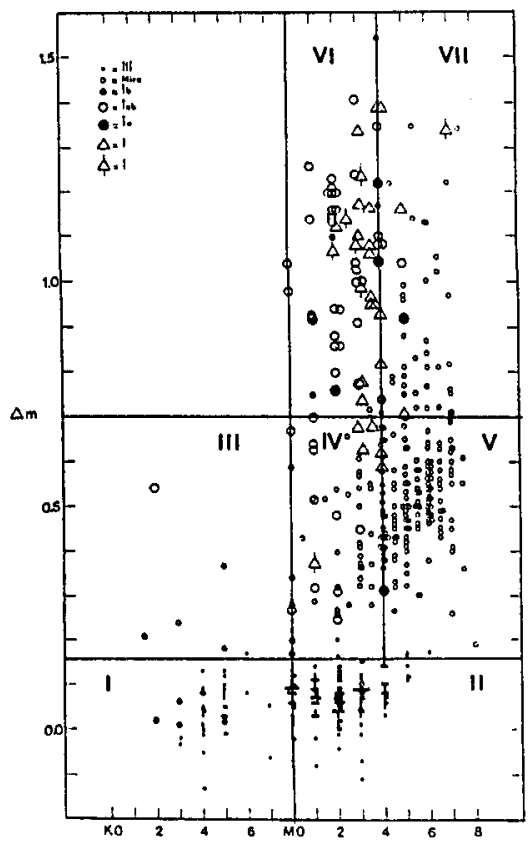

Figure 1. Infrared colour $(\Delta \mathrm{m})$ - MK spectral type diagram for $\mathrm{K}$ and $\mathrm{M}$ stars with luminosity classifications as giants and supergiants

\section{The Infrared Colour - MK Spectral Type Diagram}

The availability of extensive homogeneous infrared photometry allows us to examine the infrared colours of $M$ giants and $M$ supergiants. For this purpose, flux densities with good qualities in the IRAS 12 and $25 \mu \mathrm{m}$ bands were used. For the limiting magnitude of IRAS at 12 and $25 \mu \mathrm{m}, m_{12}=4.4$, M Miras and $M$ Supergiants can be detected to distances of 20 and $40 \mathrm{kpc}$ respectively.

The infrared colour, $m_{12}-m_{25}$, is defined as

$$
m_{12}-m_{25}=-2.5 \times \log \left(f_{l 2} / f_{25}\right)+1.56
$$

where $f_{12}$ and $f_{25}$ are the IRAS flux densities at 12 and $25 \mu \mathrm{m}$ in Jy.

Three main stellar data groups are used for this study. The ordinary $\mathrm{K} \& \mathrm{M}$ giants (238 of 243) are taken from McWilliam and Lambert (1984), Miras (222 of 228) from Keenan, Garrison and Deutsch (1974) and K \& M supergiants (86 of 91) from Elias, Frogel \& Humphreys (1985), Lee (1970), Stothers \& Leung (1971) and Buss \& Snow (1988). Most of the IRAS point source counterparts can be found easily, and have unique associations. The apparent positional differences are less than 30 arcsec. 
From the data discussed above, an infrared colour, $m_{12}-m_{25}(\Delta \mathrm{m})$, MK spectral type diagram of $K \&$ \& giant and supergiant stars was constructed. The diagram is shown in Fig. 1. Similar diagrams are given by Habing (1987a; 1987b) and Lewis (1989).

\section{Discussion}

The typical errors of IRAS flux densities for bright sources at 12 and $25 \mu \mathrm{m}$ are about 10 per cent. This will introduce a typical error in the infrared colour of about $0.2 \mathrm{mag}$. The spread in infrared colour from 0 to $\sim 1.5$ for $\mathrm{M}$ giants, $\mathrm{M}$ Miras and $M$ supergiants is significant and reflects the nature of the optical $M$ stars.

The typical error in the MK spectral classification system is less than one subclass. This is unlikely to cause a serious contamination effect in the luminosity classifications. The variability in spectral type of $M$ Miras causes changes of to up 4 subclasses which may cause a contamination effect. Since the spectral types of Miras at maximum light are used in the diagram, the spectral types at minimum light will displace the classifications towards cooler (later) spectral type.

A conversion in the $\mathrm{M}$ star classification system is necessary to avoid confusion in placing the colour-spectral type properties of $M$ stars in the colour spectral type diagram. Correlations in the spectral classification system of $\mathrm{M}$ stars are given by Mavridis (1971), Mikami (1978) and FitzGerald (1969).

It can be seen in the IR colour - MK spectral type diagram that there are clear distinct distributions for optical $M$ giants, $M$ Miras and $M$ supergiants, indicated by regions II ( $\mathrm{M} 0$ and later type with $m_{12}-m_{25}<0.15$ ), V (late $\mathrm{M}$ with $0.15<m_{12}-m_{25}<0.7$ ) and VI (early M with $0.7<m_{12}-m_{25}<1.6$ ). Region I is occupied by $\mathrm{K}$ giants and supergiants with $m_{12}-m_{25}<0.15$; region III is occupied by $\mathrm{K}$ supergiants with $0.15<m_{12}-m_{25}<0.7$. Region IV is occupied by early type $\mathrm{M}$ Miras and $\mathrm{M}$ supergiants with $0.15<m_{2}-m_{25}<0.7$. Region VII is occupied by late M Miras and M supergiants with $0.7<m_{12}-m_{25}<1.6$. It is suggested that the colour distinctions for the luminosity classifications of $\mathrm{M}$ stars in region II, V and VI can be used as an infrared colour discriminator for the luminosity class of optical M-type giant and supergiant stars.

\section{Acknowledgements}

Part of this work was done while the author visited Sterrewacht Leiden in 1984 to 1986 under an O\&W fellowship. In addition the Institute for Research, Bandung Institute of Technology provided some financial support for this work. Finally the astronomical research facilities at the National Astronomical Observatory of Japan and the Institute of Astronomy, University of Tokyo, Japan under sponsorship JSPS - DGHE were used to complete this work while the author visited Japan in 1991, 1992 and 1993. The author thanks all the above mentioned institution for providing research facilities. 


\section{References}

Albers H., 1972; ApJ, 176, 623

Beichman C. A., Neugebauer G., Habing H. J., Clegg P. E. \& Chester T. J., 1987, InfraRed Astronomical Sattelite (IRAS) Catalogue and Atlases, The Joint IRAS Science Working Group, NASA RP 1190

Buss Jr. R. H. \& Snow Jr. T. P., 1988, ApJ, 335, 331

Elias J. H., Frogel J. A. \& Humphreys R. M., 1985, ApJSS 57, 91

FitzGerald M. P., 1969, JRAS Canada 63, 251

Habing H. J., 1987a, in Circumstellar Matter IAU Symp. No 122 eds by I. Appenzeller \& C. Jordan, D. Reidel Publ., p. 197

Habing H. J., 1987b, NATO ASI Series: The Galaxy, eds G. Gilmore \& B. Carswell, D. Reidel Publ. Co. p. 173

Hacking P., Neugebauer G., Emerson J., Beichman C., Chester T., Gillett F., Habing H.J., Helou G., Houck J., Olnon F., Rowan-Robinson M., Soifer, B.T. \& Walker, D., 1985, PASP 97, 616

Ichikawa T., Hamajima K., Ishida K., Hidayat B. \& Raharto M., 1984, in Proceedings of Second IAU Asia - Pacific Regional Meeting on Astronomy, eds B. Hidayat \& M. W. Feast, Tira Pustaka, p. 117

Ishida K. \& Othman M., 1988, Astr. Sp. Sci., 148, 239

Keenan P. C., Garrison R. F. \& Deutsch A. J., 1974, ApJSS, 28, 271

Lee T. A., 1970, ApJ, 162, 217

Lewis B. M., 1989, AJ, 98, 1814

MacConnell D. J., Wing R. F. \& Costa E., 1987, Rev. Mexicana Astron. Astrophy. 14,367

Mavridis L. N., 1971, in Structure and Evolution of the Galaxy ed.L. N. Mavridis, D. Reidel, p. 110

McWilliam A. \& Lambert D. L., 1984, PASP 96, 882

Mikami T., 1978, Annals of TAO Second Ser. XVII, I

Morgan W. W. \& Keenan P. C., 1973, Ann. Rev. Astr. \& Ap., 11, 29

Nassau J. J. \& Velghe A. G., 1964, ApJ. 139, 190

Raharto M., Ishida K., Hidayat B., Ichikawa T. \& Hamajima, K., these proceedings

Stothers R. \& Leung K. C., 1971, A\&A 10, 290 